\title{
Portales educativos españoles: revisión y análisis del uso de servicios Web 2.0
}

\author{
Joaquín Martínez Méndez * \\ Francisco Javier Martínez Méndez \\ Rosana López Carreño **
}

Artículo recibido:

4 de junio de 2012.

Artículo aceptado:

24 de julio de 2012.

\section{RESUMEN}

La nueva forma de construir sitios web coloca al usuario en un lugar mucho más protagónico, de manera que puede participar de forma más activa. La Web se entiende ahora como la plataforma sobre la que se desarrollan las aplicaciones y a través de la cual los usuarios interaccionan con ella, porque las necesidades de los usuarios van más allá de la mera consulta de documentos publicados por otros autores, ahora sienten interés en editar ellos mismos sus documentos, bien de forma individual (un blog, por ejemplo) o de forma colectiva (una wiki, por ejemplo). Este nuevo paradigma se conoce con el nombre de web 2.0 e incorpora una serie de

* IES Villa de Aspe (Alicante), España.

** Los dos ultimos autores pertenecen a la Universidad de Murcia, España. (Francisco.: javima@um.es); (Rosana: rosanalc@um.es).

INVESTIGACIÓN BiBLIOTECOLÓGICA, Vol. 26, Núm. 58, septiembre/diciembre, 2012, México, ISSN: 0187-358X. pp. 47-69 
ideas y servicios que le otorgan al usuario mayor capacidad de participación, las cuales pueden ser aprovechadas de forma muy interesante por las comunidades educativas, bien de forma personal o bien apoyándose en los recursos que disponen para ello las comunidades autónomas españolas en la Web, donde desarrollan y mantienen portales que comienzan a integrar productos y servicios 2.0, que ofrecen al usuario mayor participación y personalización de su espacio personal. El objetivo de este estudio es revisar la presencia y uso de estos productos y servicios en este tipo de portales. Los resultados obtenidos nos muestran que se ha iniciado el proceso de adaptación e implementación hacia los portales educativos 2.0 pero que aún queda mucho camino por recorrer. Asimismo, y como era previsible, se han detectado grandes diferencias entre unos portales y otros en cuanto a su grado de desarrollo.

Palabras clave: Palabras clave: Web 2.0; Portal educativo; Blog; Wiki; Podcasting; Aplicaciones "en la nube"; Marcadores sociales; Redes sociales; Gestión de contenidos; Contenidos 2.0; Personalización; RSS. ${ }^{* * *}$

\section{ABSTRACT}

Spanish Educational Web portals: review and analysis of the use of Web 2.0 services.

Joaquín Martínez Méndez, Francisco Javier Martínez Méndez and Rosana López Carreño

New trends of websites construction put users in a leading role in the site development process. The Web is currently understood as the platform on which applications and services are developed allowing users interact in cyberspace above and beyond traditional query operations. Currently, users wish to edit their own contents, such as a personal blog, or as collective efforts such as a wiki. This new paradigm, known as Web 2.0, incorporates a number of innovative ideas and services that can be exploited by educational communities,

RSS Siglas que responden a Really Simple Syndication. Syndicate significa sindicar (formar parte de un sindicato, pero no es usual en nuestro español. Además en inglés syndicate tiene otro significado : "publicar artículos simultáneamente en diferentes medios a través de una fuente a la que pertenece" ( $\mathrm{N}$. del C.) 
either on one's own or through available Web resources of their Spanish autonomous communities which develop and maintain websites featuring Web 2.0 products and services. The goal of this study is to review the use of these products and services in such websites. The results obtained show that the process of adaptation and implementation of Web 2.0 has begun in these educational portals, though there is still a lot of work to be done. Moreover, as might be expected, there are significant asymmetries in the degree of development from portal to portal.

Keywords: Web 2.0; Educational portal; Blog; Wiki; Podcasting; Cloud computing applications; Social bookmarking; Social networks; Content management; Contents 2.0; Web customization; RSS.

\section{INTRODUCCIÓN}

T a forma de construir la Web ha evolucionado de manera significativa, debido a la incorporación de una serie de ideas y de tecnologías que han puesto al usuario en un lugar mucho más protagonista. Este nuevo paradigma ha recibido el nombre de Web 2.0 (O’Reilly, 2005), y su presencia es más frecuente en los portales y páginas Web. En el momento actual al usuario ya no le basta con poder acceder a la información de un sitio Web, también desea participar en la construcción del mismo.

Mediante la incorporación de los productos e ideas Web 2.0, que detallaremos y ejemplificaremos a lo largo de este trabajo, el usuario encuentra mecanismos con los cuales contribuir a la creación colaborativa de la web y, al mismo tiempo, los administradores de los portales incorporan cada vez más posibilidades de personalización de la interfaz e integran más fácilmente aplicaciones como correo electrónico, mensajería instantánea o participación en foros de debate. En definitiva el usuario construye de manera individual su espacio personal dentro del portal Web, al mismo tiempo que contribuye a la construcción colaborativa del portal. Así, esta construcción ha evolucionado no sólo por la participación en mayor o menor medida del usuario, sino porque se va abandonando la gestión estática de los mismos (la información cambia o puede cambiar rápidamente, por lo que construir y mantener de forma estática ha dejado de ser viable) y se suelen usar sistemas de gestión de contenidos o SGC (como drupal, Joomla o Wordpress) que permiten la 
habilitación de portales dinámicos y una gestión más dinámica e interactiva del portal, y facilitan su mantenimiento favoreciendo la incorporación de los productos y servicios de la Web 2.0.

Esta investigación pretende analizar los portales educativos puestos en marcha por las distintas administraciones autonómicas españolas, con el objeto de revisar si incorporan las ideas y servicios de la Web 2.0 y verificar ese grado de implantación y cuáles son sus funcionalidades y principales usos.

\section{El portal educativo}

La definición de portal Web viene dada por la concentración de información y servicios en un mismo sitio web. López Carreño (2004) afirma que se trata de un concepto mucho más amplio que el de una página o un conjunto de páginas alojadas en un servidor. La heterogeneidad de conceptos y terminologías acuñadas en torno a la Web, y más concretamente, alrededor de los portales, terminó desembocando en el desarrollo de nuevos modelos de portales, específicamente vinculados a una actividad o comunidad concreta (así se habla de portal periodístico, portal museístico, portal educativo, etcétera). Podemos definir entonces al portal educativo como aquel sitio Web donde se concentra información canalizada a través de productos y servicios relacionados con el ámbito educativo, si bien es mucho más completa la visión de Bedriñana Ascarza (2004), para quien

son espacios Web que ofrecen múltiples servicios a los miembros de la comunidad educativa (profesores, alumnos, gestores de centros y familias), tales como información, instrumentos para la búsqueda de datos, recursos didácticos, herramientas para la comunicación interpersonal, formación, asesoramiento, entretenimiento, etc.

El mismo autor (2004) expone la serie de ventajas que proporcionan a sus destinatarios estos portales, que se derivan de los servicios que ofrecen:

- Proporcionan información de todo tipo a profesores, estudiantes y padres, así como instrumentos para realizar búsquedas en Internet.

- Proporcionan recursos didácticos de todo tipo, gratuitos y utilizables directamente desde Internet (materiales didácticos en línea) o desde los ordenadores (tras «bajar» una copia desde el portal).

- Contribuyen a la formación del profesorado mediante informaciones diversas y cursos de actualización de conocimientos.

- Asesoran a los profesores. En algunos casos los portales tienen espacios 
de asesoramiento sobre diversos temas: didáctica, informática, leyes, etcétera.

- Abren canales de comunicación (foros, chats, listas...) entre profesores, estudiantes, instituciones y empresas de todo el mundo. A través de ellos se comparten ideas y materiales, se debaten temas, se consultan dudas.

- Proporcionan instrumentos para la comunicación: correo electrónico, chats, espacios para alojar páginas Web.

Debido a la gran variedad de portales educativos existentes resulta complicado clasificarlos de manera unívoca, si bien podrían clasificarse según la información tratada (generalista o específico) o el ámbito geográfico cubierto (internacional, nacional o regional). Área Moreira (2003) introduce otro aspecto claramente diferenciador: la finalidad y naturaleza del mismo, que permite identificar dos grandes grupos y a la vez dentro de cada uno de estos grupos identificar dos subgrupos principales. Así, tenemos el portal educativo informativo (Webs institucionales de consejerías, universidades, grupos de trabajo o bien un sitio Web que aloja recursos o bases de datos educativas). Por otro lado tenemos el portal educativo formativo, sitios Web creados y utilizados para generar un proceso determinado de enseñanza-aprendizaje (plataformas e-learning y sitios Web de recopilación de materiales didácticos). El mismo autor aporta un gráfico muy interesante para ilustrar esta clasificación:

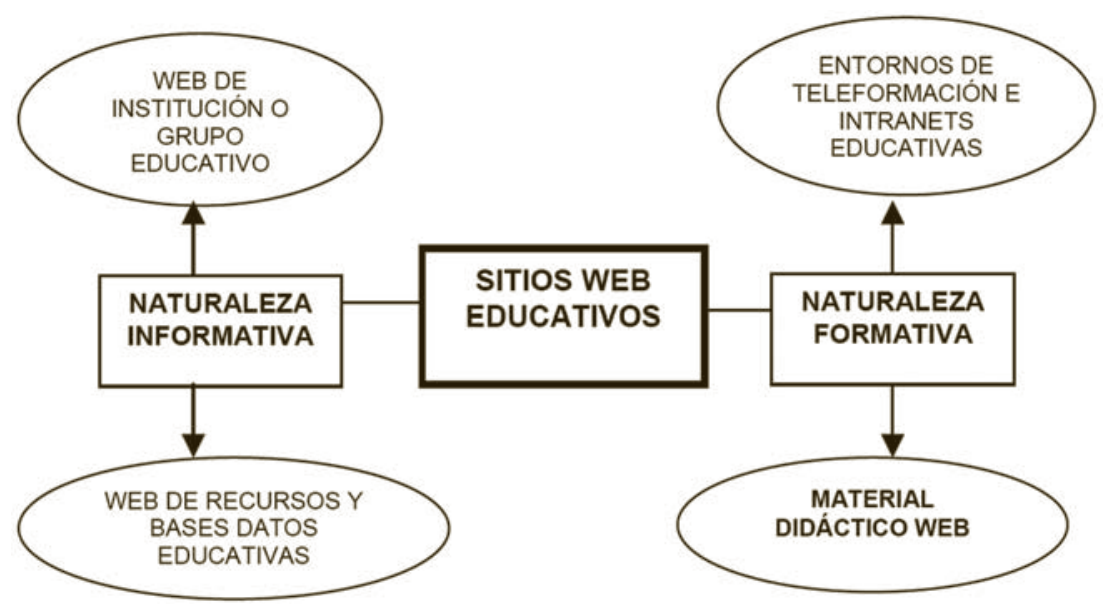

Imagen 1: Clasificación de los portales educativos.

Fuente: Área Moreira, 2003 
López Carreño (2004) avisa que "en numerosos casos podemos encontrar también, portales informativos con elementos formativos y, viceversa", por tanto si bien esta clasificación nos sirve para vehicular esta presentación de esta tipología de portales Web, no establece compartimentos estancos en modo alguno porque abundan las sinergias.

\section{Componentes de los Portales Educativos}

Al igual que ocurre en otras modalidades de portales, el contenido se constituye en la pieza clave de su diseño y funcionalidad, y es canalizado a través de los productos informativos/documentales (ítems de contenido específico y muy delimitado) y de los servicios de valor añadido (contenedores de contenido diverso y herramientas que potencian enormemente el dinamismo del portal). Estos elementos se ofertan en cualquier portal educativo (López Carreño, 2004), con independencia de su tipología (informativa o formativa):

- Productos informativos/documentales:

- Noticias o novedades

- Preguntas frecuentes

- Agenda de eventos

- Guías de recursos seleccionados: libros, Webs, etcétera

- Asesoría: didáctica, informático, legal, etcétera

- Oposiciones / bolsas de empleo

- Resúmenes o recortes de prensa

- Monográficos.

- Normativa o legislación

- Impresos o modelos

- Convocatorias / ayudas

- Estadísticas educativas

- Publicaciones

- Servicios de Valor Añadido (SVA):

La extensa gama de estos servicios es objeto de constantes cambios, por ello cualquier identificación y posterior clasificación es siempre susceptible de modificación. Se pueden agrupar en cinco categorías:

- SVA de acceso a la información: esenciales para acceder a las distintas informaciones y productos que emanan de estos portales (buscador interno, ayuda, $\mathrm{RSS}^{1}$, etcétera).

1 RSS es parte de la familia de los formatos XML desarrollado específicamente para todo tipo de sitios que se actualicen con frecuencia y por medio del cual se puede compartir la información y usarla en otros sitios web o programas. A esto se le conoce como redifusión o sindicación de contenidos. 
- SVA interactivos: permiten una interacción dinámica y frecuente entre el medio y el usuario. Están orientados a cubrir las necesidades de comunicación de los lectores (chats, foros, blogs y webmail, etcétera).

- SVA de participación: posibilitan la participación del usuario. También se produce una interacción como en el caso de los SVA Interactivos. Estos servicios están orientados a generar en el usuario un sentimiento de protagonismo propiciado por la invitación a participar en el portal (wikis, ${ }^{2}$ encuestas, etcétera).

- SVA multimedia: los orientados a reforzar la información textual, aprovechando la interacción de medios y formatos que brinda Internet (fotografías, videos, etcétera).

- SVA de formación: los pertenecientes o relacionados con el refuerzo de la formación tanto al profesorado como al alumnado; es decir, recursos educativos, materiales para estudiantes (apuntes, trabajos, exámenes...), obras de referencia atlas y mapas, tutoriales, cursos, experiencias educativas, etcétera).

Tabla 1. Clasificación de SVA en los portales educativos.

Fuente: López Carreño (2004)

\begin{tabular}{|l|l|}
\hline \multicolumn{1}{|c|}{ Categorías } & \multicolumn{1}{c|}{ SVA } \\
\hline Acceso a la información & $\begin{array}{l}\text { Ayuda, Directorio, Buscador interno, Buscador web o externo, Herramientas, } \\
\text { Mapa web, Portales verticales o temáticos, RSS, Accesibilidad }\end{array}$ \\
\hline Interactivos & $\begin{array}{l}\text { Chats, Foros, Blogs, webmail o correo electrónico web, Comunidades vir- } \\
\text { tuales }\end{array}$ \\
\hline Participación & Wikis, Encuestas, Sugerencias/Buzón \\
\hline Multimedia & Animaciones, Fotografías, Vídeos, Juegos \\
\hline Formación & $\begin{array}{l}\text { Recursos educativos, Materiales para alumnado, Obras de referencia, Atlas } \\
\text { y mapas, Tutoriales, Experiencias educativas, Encuestas de la web }\end{array}$ \\
\hline
\end{tabular}

La identificación y el análisis periódico de la presencia de estos elementos ayudan, sin duda alguna, en la revisión y valoración continua de los portales educativos, colaborando en su desarrollo. En un reciente estudio de los portales educativos institucionales dependientes de administraciones autonómicas se deduce que existe una correlación en la implantación de productos y servicios de valor añadido, hecho que se hace extensible a otras modalidades de portales, potenciado por el interés de equilibrar los elementos básicos de los portales (productos y servicio) para proporcionar un mayor grado de calidad

2 Un wiki es un sistema de publicación en la web que se caracteriza porque cualquiera puede modificar una página. Permiten crear colectivamente documentos web, sin que la revisión del contenido sea necesaria antes de su aceptación para ser publicado en la red. Los más famosos son las enciclopedias colaborativas libres en línea (Wikipedia es el ejemplo más conocido). 
en la oferta de sus contenidos. Por otra parte, los servicios correspondientes a la mejora del acceso a la información y a la formación son los que se presentan con una mayor implantación en los portales indicados debido, por un lado, al amplio espectro de usuarios con necesidades informativas diversas y, por el otro, a la implicación instructiva a la que se tiende en cualquier tipología de portal educativo. Los servicios de blog, wiki y webquest se proyectan como elementos indispensables a corto plazo en el desarrollo de portales educativos debido a sus cualidades didácticas intrínsecas que se presentan, no sólo como elementos complementarios en los procesos actuales de enseñanza-aprendizaje, sino también como herramientas que fomentan la construcción social del conocimiento y la inteligencia colectiva (Lara, 2008).

\section{La Web 2.0}

La evolución de la web en los últimos años no ha sido únicamente tecnológica sino también sociológica. La mejora progresiva de las infraestructuras de acceso a Internet (mayores anchos de banda) y su cada vez mejor acceso desde multiplataformas (ordenadores o computadoras, netbooks, smartphones, Asistente Personal Digital (APD), tablets y TV por Internet) viene acompañado ineludiblemente de un número mayor de usuarios y, además, de nuevas maneras de entender la Web (e Internet en general) como medio de comunicación social. La Web se transforma de forma paulatina, los usuarios van más allá de consultar documentos publicados por otros, ahora sienten interés en editar ellos mismos sus documentos, bien de forma individual (un blog, por ejemplo) o de forma colectiva (una wiki). Ahora mismo, la Web ya no es sólo un espacio de difusión de información, es algo mucho más dinámico y participativo porque los usuarios interaccionamos de forma continua con ella bien adquiriendo productos o servicios (banca o comercio electrónico) o bien dándoles forma a nuestros propios espacios en forma de colecciones de fotos, vídeos o comentarios. Igualmente propiciamos el intercambio de ideas y la promoción de actividades vía redes sociales, no limitándonos a la publicación de comentarios entre grupos de amigos sino también facilitando la creación y difusión de nuevo conocimiento de forma compartida, tal como sucede con los wikis (con Wikipedia como ejemplo paradigmático). Este proceso de creación dinámica de contenidos, la mayoría de ellos de acceso abierto, tiene su reflejo en el desarrollo colaborativo de aplicaciones de software libre por medio de comunidades de informáticos que las brindan en acceso gratuito. Con esta integración de comunidades y voluntades podemos decir que en el momento actual la web está adquiriendo ese carácter universal al que aludía Berners-Lee en su origen (1989). 
El establecimiento de comunidades de usuarios altamente participativos tiene una faceta social, que define claramente el actual paradigma Web 2.0 (también llamada "Web Social"). De hecho, este nombre surge en el seno de una "tormenta de ideas" coordinada por el consultor Dale Daugherty y desarrollada por medio de una discusión en línea. Web 2.0 es la transición que se ha dado de aplicaciones tradicionales hacia aplicaciones que funcionan a través de la red (se pasa del escritorio del ordenador o computadora al escritorio alojado en un sitio Web junto con una serie de aplicaciones ofimáticas compartidas tipo Google Calendar o Microsoft Word Windows Live), enfocadas hacia el usuario final. Asimismo, un portal web ya no se construye y mantiene de forma estática por medio de editores de páginas web (tipo $M i$ crosoft Frontpage o Adobe Dreamweaver), ahora los portales web se diseñan con los sistemas de gestión de contenidos o SGC (GMS) (tipo drupal o Joomla) que permiten la habilitación de portales dinámicos. Al mismo tiempo que se produce un avance tecnológico, se da un cambio de actitud desde el punto de vista del usuario: la idea subyacente es simple, la web tradicional venía a ser un sistema "de sólo lectura", la Web 2.0 es un contexto "lectura/escritura" desarrollado alrededor de nuevas ideas abstractas que definen tendencias a posteriori -entre las que destacan convergencia, remezclabilidad, estandarización, usabilidad y participación- fusionadas con el desarrollo de nuevas tecnologías (videocasting, XHTML, SOAP, podcasting, agregación por (RSS), etcétera). y servicios (microformatos, audio, movilidad, wikis, folksonomías). La siguiente imagen es un mapa mental que ofrece una perspectiva más global de lo que se entiende por Web 2.0.

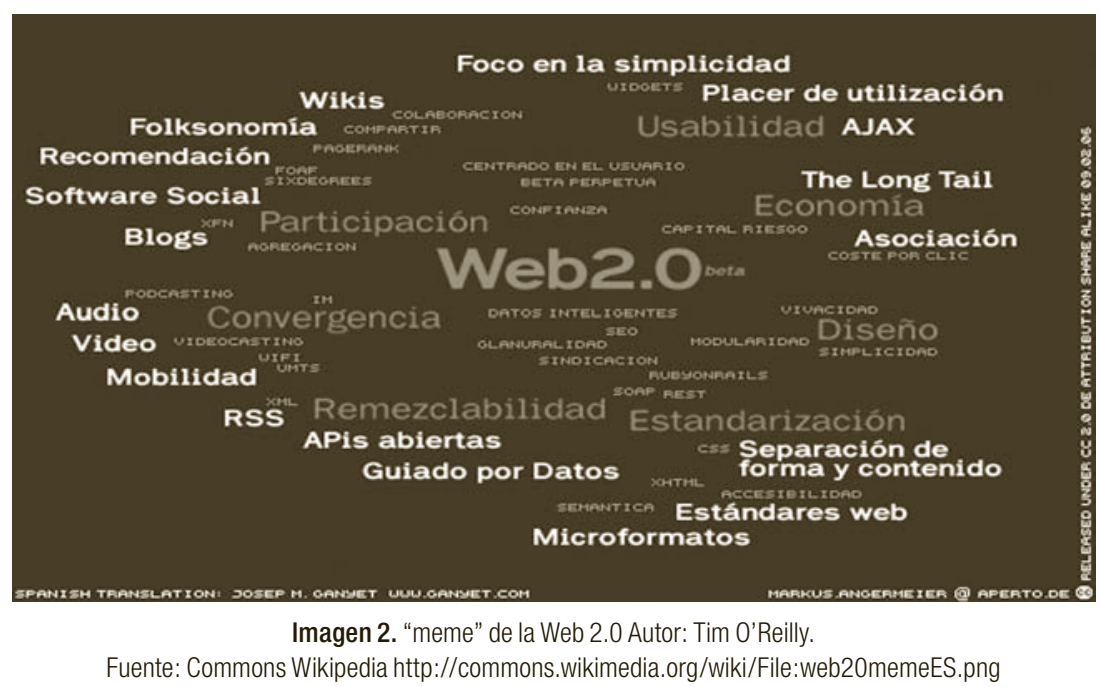


Centrándonos en el área superior izquierda de este mapa mental, la parte más relacionada con la Información y la Comunicación (el área de la Participación), destacan los siguientes servicios:

a) Wikis: sitio web cuyas páginas web pueden ser editadas por múltiples voluntarios a través del navegador web compartiendo el acceso y la autoridad del documento. Los textos o "páginas wiki" tienen títulos únicos. La aplicación más conocida y a la que debe esta tecnología su fama es la enciclopedia colaborativa Wikipedia, ideada por 'Jimbo' Wales. Existen muchas otras aplicaciones más cercanas a la coordinación de informaciones y acciones, o la puesta en común de conocimientos o textos dentro de grupos.

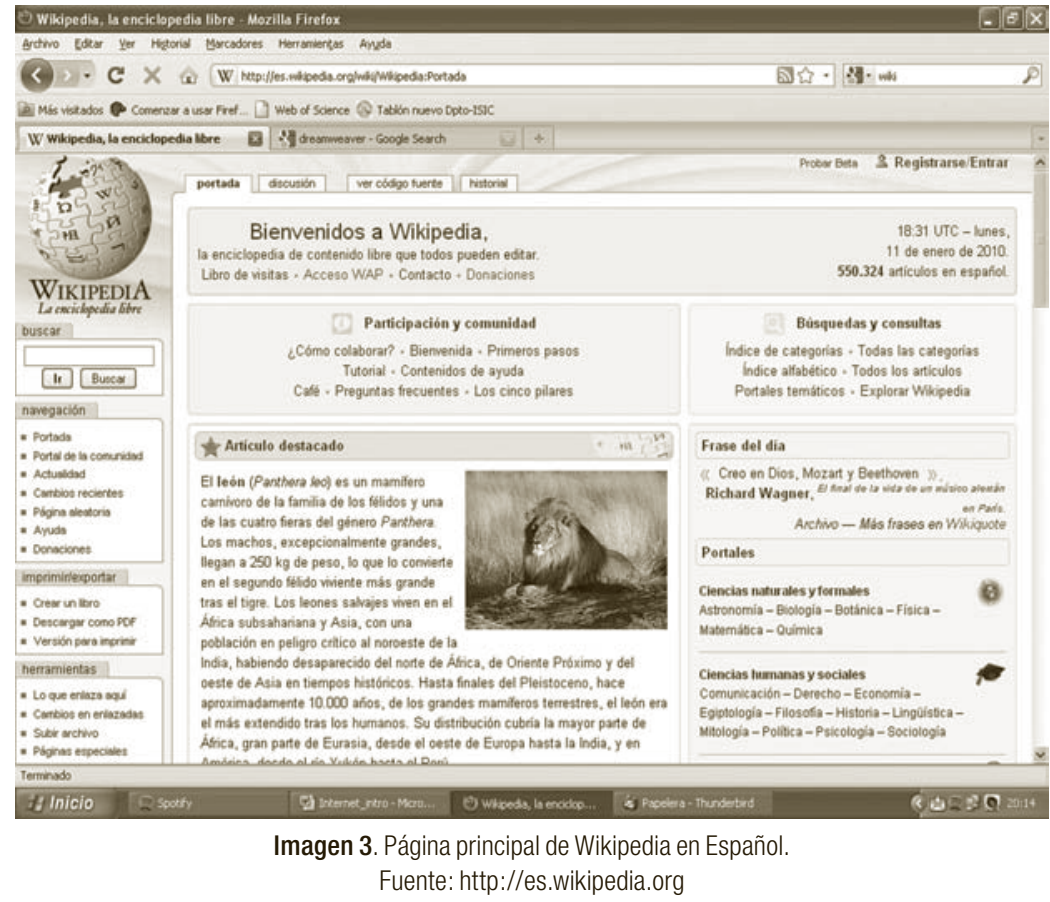

b) Folksonomias: son sistemas de etiquetado libre que permiten una clasificación grupal de los contenidos, de manera que se genera una taxonomía social u organización de la información colectiva, basada en la cooperación de las personas que ordenan contenidos mediante etiquetas. Las categorías usadas no suelen obedecer a una lógica jerárquica, como ocurre en las clasificaciones bibliotecarias, sino a las decisiones de etiquetado de los usuarios. 
c) Software social: este concepto se refiere a los sistemas que favorecen la integración de las personas, la información, el trabajo y la tecnología en una dinámica constructiva. En la web tradicional ya se desarrollaron portales de redes de contactos personales (match.com o meetic.com por ejemplo), servicios generalmente de pago. En la Web 2.0 las redes sociales se han abierto mucho más para intercambiar ideas, comentarios y fomentar relaciones personales (familiares, grupos de amigos, vecinos, etc.), especialmente tras el éxito de Facebook (la red social más conocida que surgió en el seno de la Universidad de Harvard con el propósito de poner en contacto a los residentes del campus), aunque no es la única (también está tuenti muy popular entre los jóvenes españoles y la reciente Google+). Igualmente hay redes sociales dirigidas a poner en contacto a profesionales vinculados a un sector laboral (linkedln o academia.edu) y también de citas de recursos de investigación como es el caso de Google Citations o Microsoft Academic Search. Una razón del auge de estas redes sociales es su alta capacidad de integración con otras fuentes 2.0 tales como videos o noticias de portales de medios de comunicación o comentarios realizados en los blogs. No sólo las redes sociales entran dentro de este apartado, también hay que considerar los marcadores sociales, el etiquetado social y los buscadores sociales, sistemas que permiten construir una inteligencia colectiva y acceder al conocimiento almacenado por vías alternativas al uso de los tradicionales motores de búsqueda o directorios.

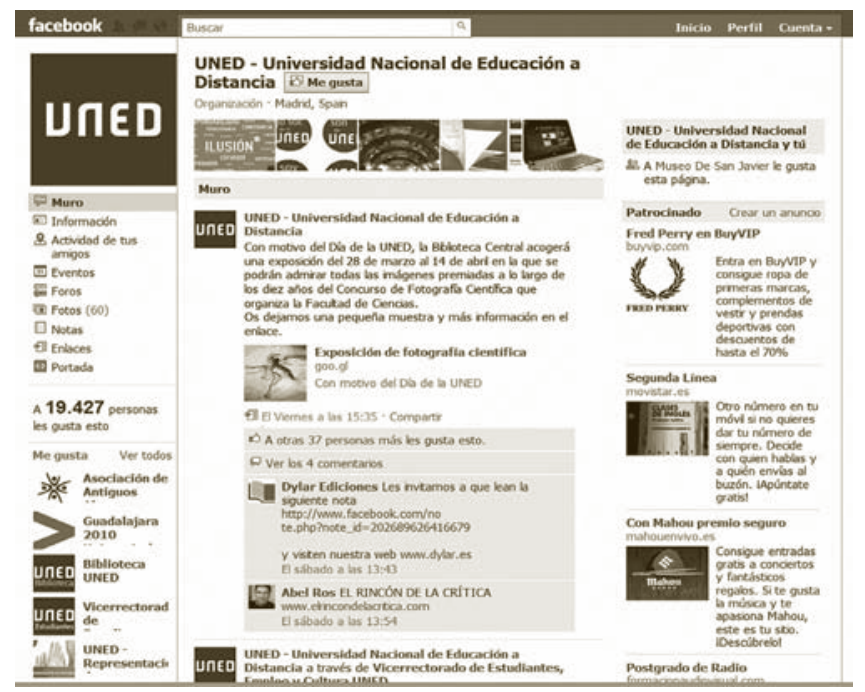

Imagen 4. Muro en Facebook de la UNED (Universidad Nacional de Educación a Distancia). Fuente: http://facebook.com 
d) Blogs (o cuaderno de bitácora): sitio web periódicamente actualizado que recopila cronológicamente textos o artículos de uno o varios autores, apareciendo primero el más reciente. Habitualmente, los lectores pueden escribir sus comentarios y el autor les contesta, estableciendo un diálogo. Hay una gran variedad de usos y temas: reflexiones y vivencias personales, informativos, corporativos, tecnológicos, educacionales (edublogs), políticos, etc. Existen también variedades de este formato, conocidas como fotoblogs (sitios que publican unas pocos fotos al día y se hacen comentarios), los videoblogs (galería de clips de vídeos, ordenada cronológicamente, publicados por uno o más autores). El autor puede autorizar a otros usuarios a añadir comentarios u otros vídeos en la misma galería). Los contenedores de blogs más utilizados son blogger.com y Wordpress. com si bien es posible alojar un blog en un portal, como ocurre en Educastur para desarrollar una comunidad de blogs educativos.

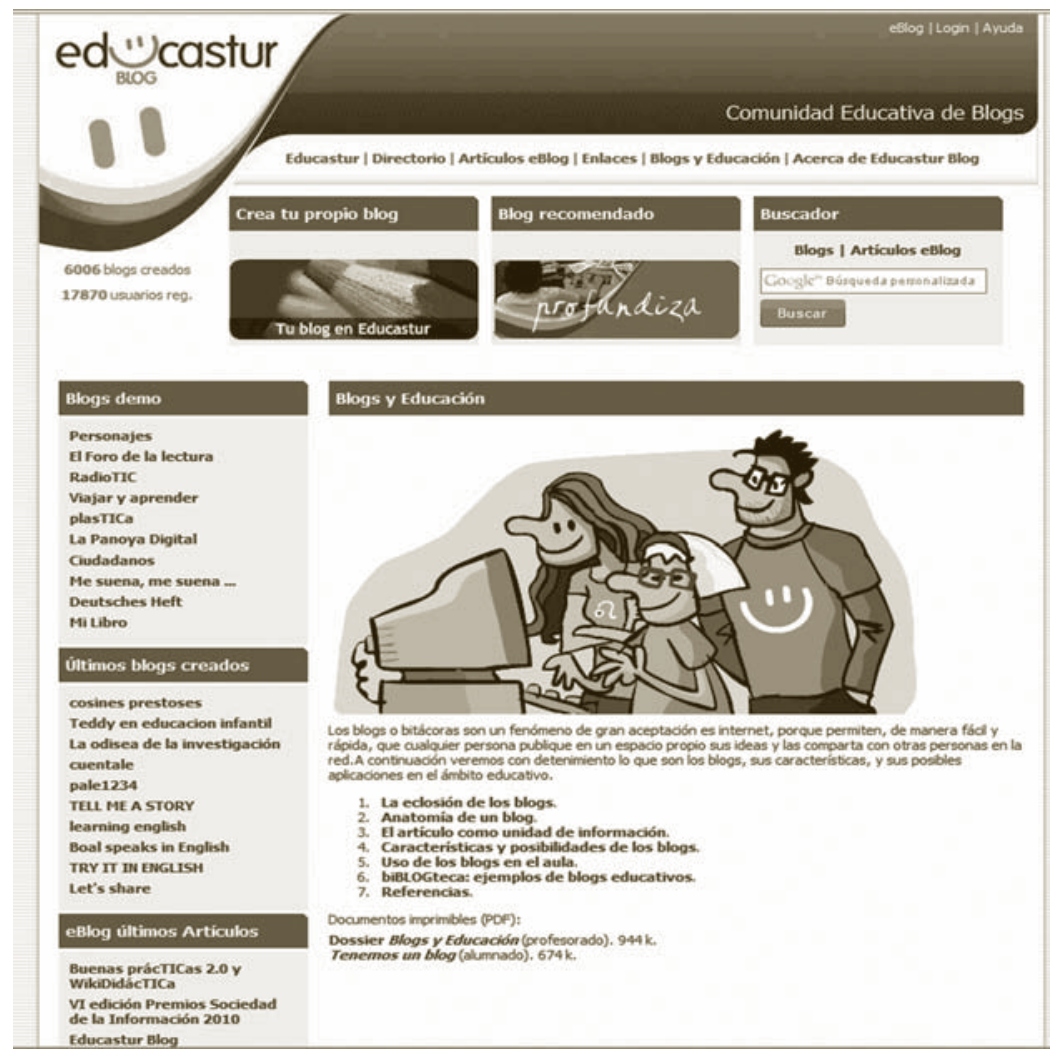

Imagen 5. Comunidad educativa de blogs del portal Educastur.

Fuente: http://blog.educastur.es/blogs-y-educacion/ 
A medias entre el software social y los blogs se halla el concepto de microblogging puesto de moda por twitter. Es un servicio gratuito para el envío de mensajes de texto ("tweets") con longitud máxima de 140 caracteres y que pueden ser publicadas tanto en la cuenta del usuario en twitter como en otros servicios sociales al mismo tiempo que se permite su reenvío vía SMS o correo electrónico.

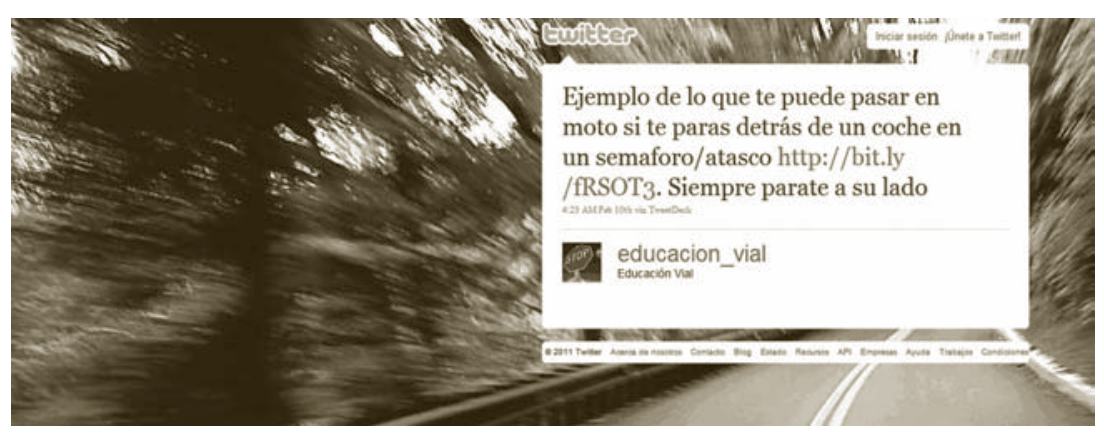

Imagen 6: Uso de twitter dentro de un programa formativo sobre Educación Vial. Fuente: http://twitter.com/educacion_vial/status/35675124438933504

Esta aplicación podría parecer poco relevante en usos educativos, pero no es así del todo. Wheeler (2009) define hasta diez posibles usos, entre los que destacan: tablón de anuncios, aprender a resumir y a compartir enlaces de forma habitual, el seguimiento de un personaje famoso y la posibilidad de "rescatar del pasado" a personajes históricos asumiendo su rol actual, elaboración de microrrelatos, traducciones de términos y aprendizaje de nuevas culturas o lugares geográficos por medio de conversaciones mantenidas con otras personas.

\section{OBjetivos y METODOLOGÍA}

Una vez establecido el marco general de la investigación, la Web 2.0, queda por definir el objeto de la misma: ponderar debidamente su presencia e inserción entre los distintos portales educativos desarrollados por las distintas comunidades autónomas, organismos competentes en Educación en aplicación de sus respectivos estatutos de autonomía. Nuestra atención se centra en los portales educativos que ofrecen y alojan servicios para la comunidad educativa, algo que bien puede llevar a cabo el portal institucional administrativo (las menos veces) como un portal educativo desarrollado ad-hoc con este propósito. Lo primero fue identificar los portales educativos de las distintas 
administraciones y luego discernir cuáles cumplían con nuestro requisito. Finalmente se seleccionaron 19 portales educativos (17 de las comunidades y 2 de las ciudades autónomas): averroes (Andalucía); educaragón (Aragón); educantabria (Cantabria); educacyl (Castilla y León); Portal educativo Castilla-LM; EDU365.cat (Cataluña); Portal educativo de Ceuta; Portal Educativo de Melilla; EDUCAMADRID (Madrid); Programa para las Nuevas Tecnologías y Educación, PNTE (Navarra); mestre@casa (C. Valenciana); edecarEx (Extremadura); Espazo Abalar (Galicia); Informática educativa (I. Baleares); ecosecuela 2.0 (I. Canarias); educarioja.org (La Rioja); Eskola 2.0 (País Vasco); Educastur (Asturias) y Aula XXI (Murcia). El periodo de revisión de estos portales fue el comprendido entre marzo y mayo del año 2011.

En la revisión se consideraron 31 productos o servicios 2.0 a partir del famoso "meme" de la Web 2.0 de O'Reilly (ver Imagen 6) desarrollado en el libro "La web 2.0" de la Fundación Orange (2007). 
Tabla 2. Servicios y aplicaciones de las web 2.0 estudiados en los los portales educativos.

Fuente: elaboración propia

\begin{tabular}{|c|c|}
\hline $\begin{array}{l}\text { Páginas de inicio personalizadas. } \\
\text { Blogs. } \\
\text { Sistemas de creación y alojamiento. } \\
\text { Ranking de blogs y herramientas. } \\
\text { Comunidades, directorios de blogs. } \\
\text { Redes de blogs. } \\
\text { Podcasting. } \\
\text { Audio. } \\
\text { Videoblogs. } \\
\text { Wikis. } \\
\text { Consulta. } \\
\text { Edición. } \\
\text { Aplicaciones "en la nube". } \\
\text { Correo electrónico. } \\
\text { Mensajería. } \\
\text { Foros de debate. } \\
\text { Ofimática } 2.0 .^{6} \\
\text { Redes sociales. } \\
\text { Personales/Generalistas. } 7 \\
\text { Profesionales. }\end{array}$ & $\begin{array}{l}\text { Marcadores sociales. } \\
\text { Almacenamiento direcciones. } \\
\text { Etiquetado ('folksonomías' }{ }^{3} \text { ). } \\
\text { Nubes de etiquetas ('tag clouds' }{ }^{4} \text { ). } \\
\text { Multimedia. } \\
\text { Compartir fotos. } \\
\text { Compartir vídeos. } \\
\text { Distribución canales de video. } \\
\text { Comunidades móviles. } \\
\text { Sistemas de gestión de contenidos. } \\
\text { Creación de 'webquests'. } \\
\text { Edición de contenidos educativos. } \\
\text { Edición y realización de cursos } \\
\text { MLCC Métodos Lógicos en Ciencia } \\
\text { de la Computación o LMCS. } \\
\text { Contenidos } 2.0 \text {. } \\
\text { Buscadores } 2.0 \\
\text { Recomendaciones de contenidos. } \\
\text { Votar noticias y contenidos. } \\
\text { Agregadores/lectores RSS de con- } \\
\text { tenido. } \\
\text { Lectores RSS. } \\
\text { Aplicaciones sobre mapas. }\end{array}$ \\
\hline
\end{tabular}

La siguiente tabla muestra los portales identificados y los analizados en cada caso:

3 Folksonomía es una indexación social o una clasificación colaborativa por medio de etiquetas simples en un espacio de nombres llano, sin jerarquías predeterminadas. Es una práctica típica en entornos de marcadores o etiquetado social cuyos exponentes son del.icio.us (enlaces favoritos) o Flickr (fotos).

4 Sistema de visualización de la información del portal a partir de una nube de etiquetas definidas por los creadores de contenidos y de una serie de folksonomías.

5 Archivos de sonido distribuidos por suscripción. Pueden ser un apoyo para actividades docentes.

6 Acceso a editor de texto, agenda de direcciones, gestor de base de datos y otras aplicaciones ofimáticas instaladas en entorno de red ("nube").

7 Una red social personal o generalista es aquella que está abierta en principio para todo el mundo. Una red profesional es la creada pensando en conectar colectivos específicos de trabajadores.

8 El portal genera una fuente informativa RSS que puede leerse por lectores RSS, clientes de correo o bien el portal habilita una zona de lectura de fuentes RSS externas (como hace bloglines). 
Tabla 3. Portales educativos de las CC.AA. y clasificación.

Fuente: elaboración propia.

\begin{tabular}{|c|c|c|c|}
\hline Comunidad & Institucional & Educativo-Informativo & Educativo-Formativo \\
\hline Andalucía & $\begin{array}{l}\text { Junta de Andalucía. } \\
\text { Consejería de Educación. }\end{array}$ & & averroes \\
\hline Aragón & $\begin{array}{l}\text { Gobierno de Aragón. } \\
\text { Educación. }\end{array}$ & educaragón & \\
\hline Cantabria & $\begin{array}{l}\text { Gobierno de Cantabria. } \\
\text { Educación. }\end{array}$ & educantabria & \\
\hline Castilla y León & & educacyl & \\
\hline Castilla y La Mancha & $\begin{array}{l}\text { Junta de Castilla y La } \\
\text { Mancha. Consejería de } \\
\text { Educación. }\end{array}$ & $\begin{array}{l}\text { Portal de educación de } \\
\text { Castilla y la Mancha }\end{array}$ & \\
\hline Cataluña & $\begin{array}{l}\text { Generalitat de Cataluña. } \\
\text { Departamento de } \\
\text { Enseñanza. }\end{array}$ & $\begin{array}{l}\text { Red telemática educativa } \\
\text { de Cataluña (xtec) }\end{array}$ & EDU365.cat \\
\hline $\begin{array}{l}\text { Ciudad Autónoma de } \\
\text { Ceuta }\end{array}$ & $\begin{array}{l}\text { Ciudad Autónoma de } \\
\text { Ceuta. Consejería de } \\
\text { Educación. }\end{array}$ & & \\
\hline $\begin{array}{l}\text { Ciudad Autónoma de } \\
\text { Melilla }\end{array}$ & $\begin{array}{l}\text { Ciudad Autónoma de } \\
\text { Melilla. Consejería de } \\
\text { Educación. }\end{array}$ & & \\
\hline Comunidad de Madrid & $\begin{array}{l}\text { Comunidad de Madrid. } \\
\text { Consejería de Educación }\end{array}$ & +educación & EDUCAMADRID \\
\hline $\begin{array}{l}\text { Comunidad Foral de } \\
\text { Navarra }\end{array}$ & $\begin{array}{l}\text { Gobierno de Navarra. } \\
\text { Departamento de } \\
\text { Educación. }\end{array}$ & $\begin{array}{l}\text { Portal educativo del De- } \\
\text { partamento de Educación }\end{array}$ & $\begin{array}{l}\text { Programa de Nuevas } \\
\text { Tecnologías y Educación } \\
\text { (PNTE) }\end{array}$ \\
\hline Comunidad Valenciana & & $\begin{array}{l}\text { Generalitat Valenciana. } \\
\text { Consejería de Educación. }\end{array}$ & mestre@casa \\
\hline Extremadura & $\begin{array}{l}\text { Junta de Extremadura. } \\
\text { Consejería de Educación. }\end{array}$ & educarEx & $\begin{array}{l}\text { contenidos EDUCATIVOS } \\
\text { DIGITALES }\end{array}$ \\
\hline Galicia & $\begin{array}{l}\text { Xunta de Galicia. Conse- } \\
\text { jería de Educación. }\end{array}$ & & Espazo Abalar \\
\hline Islas Baleares & $\begin{array}{l}\text { Govern de Illes Balears. } \\
\text { Consejería de Educación. }\end{array}$ & $\begin{array}{l}\text { El web educativo de Illes } \\
\text { Balears (weib) }\end{array}$ & informàtica educativa \\
\hline Islas Canarias & $\begin{array}{l}\text { Gobierno de Canarias. } \\
\text { Consejería de Educación. }\end{array}$ & & Portal Medusa \\
\hline La Rioja & $\begin{array}{l}\text { Gobierno de la Rioja. } \\
\text { Área de Educación }\end{array}$ & eudcarioja.org & \\
\hline País Vasco & & $\begin{array}{l}\text { Gobierno del País } \\
\text { Vasco. Departamento de } \\
\text { Educación. }\end{array}$ & Eskola 2.0 \\
\hline Principado de Asturias & $\begin{array}{l}\text { Gobierno del Principado. } \\
\text { Consejería de Educación. }\end{array}$ & Educastur & \\
\hline Región de Murcia & $\begin{array}{l}\text { Región de Murcia. Con- } \\
\text { sejería de Educación. }\end{array}$ & Educarm & Aula XXI \\
\hline
\end{tabular}




\section{DisCUSión DE RESUlTADOS}

En la siguiente tabla se muestra un resumen de los resultados obtenidos tras aplicar la guía de observación que nos ha permitido distinguir la presencia de los servicios y aplicaciones de la Web 2.0 en los 19 portales educativos analizados. En primer lugar se indica para los portales evaluados, cuántos y cuáles son de tipo institucional, de tipo informativo y de tipo formativo.

Tabla 4. Estadística sobre el número de portales analizados de cada tipo.

\begin{tabular}{|c|c|c|c|c|c|c|}
\hline \multicolumn{7}{|c|}{ Número de portales de cada tipo } \\
\hline \multicolumn{2}{|c|}{ Tipo Institucional } & \multicolumn{2}{|c|}{ Tipo Informativo } & \multicolumn{2}{|c|}{ Tipo Formativo } & \multirow[t]{4}{*}{ Gráfico } \\
\hline Número & Porcentaje & Número & Porcentaje & Número & Porcentaje & \\
\hline 2 & $10,53 \%$ & 7 & $36,84 \%$ & 10 & $52,63 \%$ & \\
\hline \multicolumn{2}{|c|}{ Nombre de los portales } & \multicolumn{2}{|c|}{ Nombre de los portales } & \multicolumn{2}{|c|}{ Nombre de los portales } & \\
\hline \multicolumn{2}{|c|}{$\begin{array}{l}\text { Portal educativo Ceuta } \\
\text { Portal Educativo Melilla }\end{array}$} & \multicolumn{2}{|c|}{$\begin{array}{l}\text { educaragón (Aragón) } \\
\text { educantabria (Cantabria) } \\
\text { educacyl (Castilla y León) } \\
\text { Portal educativo Castilla-LM } \\
\text { edecarEx (Extremadura) } \\
\text { educarioja.org (La Rioja) } \\
\text { Educastur (Asturias) }\end{array}$} & \multicolumn{2}{|c|}{$\begin{array}{l}\text { averroes (Andalucía) } \\
\text { EDU365.cat (Cataluña) } \\
\text { EDUCAMADRID (Madrid) } \\
\text { PNTE (Navarra) } \\
\text { mestre@casa (C. Valen- } \\
\text { ciana) } \\
\text { Espazo Abalar (Galicia) } \\
\text { Informática educativa (I. } \\
\text { Baleares) } \\
\text { ecosecuela 2.0 (I. Canarias) } \\
\text { Eskola 2.0 (País Vasco) } \\
\text { Aula XXI (Murcia) }\end{array}$} & $\begin{array}{l}\text { - Institucionales } \\
\text { - Informativos } \\
\text { w Formativos } \\
\begin{array}{c}10,53 \\
\%\end{array} \\
2,63\end{array}$ \\
\hline
\end{tabular}

En la Tabla 5 (página siguiente) se recoge un "ranking" de los productos y servicios de la Web 2.0 considerados en esta investigación ordenado según el número de portales que los incorporan (de mayor a menor). 


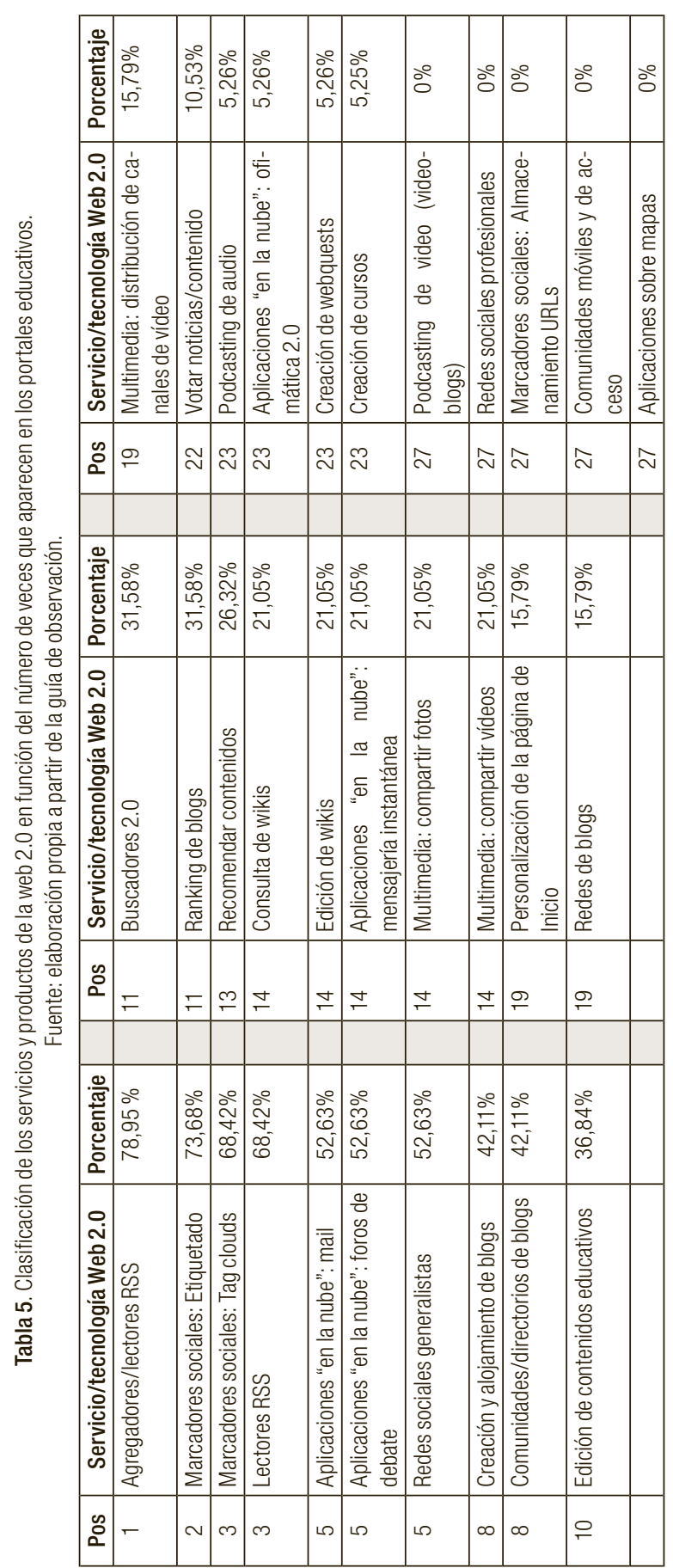


Los datos de la tabla anterior reflejan que existe una gran dispersión entre unos servicios y otros, algo que responde al nivel de desarrollo de los portales (es más fácil habilitar la distribución de información vía RSS que viene casi por defecto implementada por el SGC, Sistemas de Gestión de Contenidos o CMS, que habilitar el acceso a aplicaciones "en la nube"). En la siguiente tabla se muestra esta información de manera más detallada:

Tabla 6. Clasificación de los portales educativos en función del número de servicios y productos 2.0 que integran. Fuente: elaboración propia a partir de la guía de evaluación.

\begin{tabular}{|c|c|c|}
\hline $\begin{array}{c}\text { Portal } \\
\text { Educativo }\end{array}$ & $\begin{array}{l}\text { Núm. de } \\
\text { elementos } \\
\text { Web } 2.0\end{array}$ & Comentarios \\
\hline $\begin{array}{l}\text { EDUCAMADRID } \\
\text { (Madrid) }\end{array}$ & $17-58,84 \%$ & $\begin{array}{l}\text { Apuesta por el aprendizaje colaborativo. El usuario asume un papel } \\
\text { más importante en la elaboración del portal. }\end{array}$ \\
\hline $\begin{array}{l}\text { EDU365.cat } \\
\text { (Cataluña) }\end{array}$ & $17-58,84 \%$ & $\begin{array}{l}\text { La integración de las filosofías Web } 2.0 \text { y sus tecnologías es cierta- } \\
\text { mente significativa. Se encuentra enlazado con los portales XTEC y } \\
\text { EDU3.cat dependientes de la Generalitat y algunos elementos que no } \\
\text { se encuentran en este portal sí pueden aparecer en los otros. }\end{array}$ \\
\hline $\begin{array}{l}\text { educarEx } \\
\text { (Extremadura) }\end{array}$ & $14-45,16 \%$ & $\begin{array}{l}\text { Conjunción de otros portales educativos: mercurio (multimedia), Pro- } \\
\text { fex (comunicación entre docentes), enmarchaconlastics (portal 2.0) } \\
\text { y contenidos educativos digitales (repositorio materiales). Moderada } \\
\text { presencia de tecnologías 2.0. Gran dispersión de contenidos. }\end{array}$ \\
\hline $\begin{array}{l}\text { Educaragón } \\
\text { (Aragón) }\end{array}$ & $12-38,71 \%$ & $\begin{array}{l}\text { A medio camino entre institucional y educativo (tipo formativo). Se } \\
\text { apoya en el portal catedu (recursos didácticos y herramientas de edi- } \\
\text { ción) y en el directorio "ArablogsBeta". Usuario activo en la construc- } \\
\text { ción del portal mediante blogging. Ofrece proyectos como Escuela } \\
2.0\end{array}$ \\
\hline PNTE (Navarra) & $12-38,71 \%$ & $\begin{array}{l}\text { Portal educativo formativo. Integra algunas tecnologías y contenidos } \\
\text { 2.0. Presencia importante de blogs, foros y etiquetado. Ofrece sindi- } \\
\text { cación RSS y servicios ofimáticos para usuarios registrados. Partici- } \\
\text { pación activa del usuario en la construcción. Faltan elementos impor- } \\
\text { tantes. Aún puede incorporar muchas ideas y servicios } 2.0\end{array}$ \\
\hline $\begin{array}{l}\text { Aula XII (Región } \\
\text { de Murcia) }\end{array}$ & $11-35,48 \%$ & $\begin{array}{l}\text { Es un buen intento de desarrollo de un portal educativo } 2.0 \text { aunque } \\
\text { aún son muchos los aspectos a mejorar. Los docentes disponen ya de } \\
\text { un número importante de herramientas 2.0. Falta dinamización del } \\
\text { portal, los usuarios también tienen algo importante que decir y que } \\
\text { aportar. }\end{array}$ \\
\hline $\begin{array}{l}\text { averroes } \\
\text { (Andalucía) }\end{array}$ & $10-32,36 \%$ & $\begin{array}{l}\text { Ofrece algunos servicios informativos, si bien es principalmente for- } \\
\text { mativo. Incorpora participación del usuario en construcción (blogging } \\
\text { y redes sociales). También hay sindicación RSS y aplicaciones "en la } \\
\text { nube" para usuarios registrados. Se encuentra aún algo lejos de la } \\
\text { filosofía 2.0, y faltan mecanismos para que el usuario intervenga de } \\
\text { forma más activa. }\end{array}$ \\
\hline $\begin{array}{l}\text { Informática } \\
\text { Educativa (Islas } \\
\text { Baleares) }\end{array}$ & $10-32,36 \%$ & $\begin{array}{l}\text { Carece de la mayoría de elementos } 2.0 \text { analizados en esta investi- } \\
\text { gación. Ofrece principalmente acceso a recursos educativos, pero } \\
\text { no dispone acceso del usuario a elementos para que participe en la } \\
\text { construcción del portal. Incorpora sindicación RSS. Es más bien un } \\
\text { portal Web tradicional. }\end{array}$ \\
\hline
\end{tabular}




\begin{tabular}{|c|c|c|}
\hline $\begin{array}{l}\text { educastur } \\
\text { (Asturias) }\end{array}$ & $10-32,36 \%$ & $\begin{array}{l}\text { A medio camino entre portal institucional y educativo tipo formativo. } \\
\text { Hay información institucional, noticias y utilidades de interés para la } \\
\text { comunidad educativa. El portal carece de muchos elementos de la } \\
\text { WEB } 2.0\end{array}$ \\
\hline $\begin{array}{l}\text { eco escuela } 2.0 \\
\text { (Islas Canarias) }\end{array}$ & $9-29,93 \%$ & $\begin{array}{l}\text { Importante orientación hacia la Web } 2.0 \text { si bien carece de algunas } \\
\text { características destacadas como personalización de la página de } \\
\text { inicio, podcasting, aplicaciones en la "nube", compartición de mul- } \\
\text { timedia y algunos aspectos sobre gestión de contenidos, entre otros. }\end{array}$ \\
\hline $\begin{array}{l}\text { Educantabria } \\
\text { (Cantabria) }\end{array}$ & $9-29,93 \%$ & $\begin{array}{l}\text { Incorpora algunos elementos y productos } 2.0 \text { para proporcionarle un } \\
\text { espacio personal al usuario, que colabora en la construcción. Perso- } \\
\text { nalización de la interfaz y edición de contenidos educativos, además } \\
\text { de algunos servicios como mail, foros y sindicación RSS. El camino } \\
\text { por recorrer es aún grande. }\end{array}$ \\
\hline $\begin{array}{l}\text { Portal de } \\
\text { Castilla-LM }\end{array}$ & $8-25,81 \%$ & $\begin{array}{l}\text { Portal educativo informativo que incorpora algunos principios y tec- } \\
\text { nologías } 2.0 \text { aunque aún tiene un largo camino por recorrer. Ofrece } \\
\text { sindicación RSS y presencia de redes sociales, blogs educativos. El } \\
\text { usuario no tiene protagonismo en la construcción colaborativa del } \\
\text { portal. }\end{array}$ \\
\hline $\begin{array}{l}\text { mestre@casa } \\
\text { (C. Valenciana) }\end{array}$ & $6-19,35 \%$ & $\begin{array}{l}\text { Portal educativo formativo con escasas ideas y funciones 2.0. Herra- } \\
\text { mienta para docentes y comunidad educativa en general con algunos } \\
\text { recursos educativos. En la parte privada (acceso autenticado) ofrece } \\
\text { aplicaciones "en la nube" (mail, chat y foros) únicos signos junto con } \\
\text { elaborar y consultar una wiki de la Web Social. El usuario apenas par- } \\
\text { ticipa. }\end{array}$ \\
\hline $\begin{array}{l}\text { skola } 2.0 \\
\text { (País Vasco) }\end{array}$ & $6-19,35 \%$ & $\begin{array}{l}\text { Portal educativo-formativo diseñado como repositorio de recursos } \\
\text { educativo-didácticos muy útiles para formadores. Se enlazan gran } \\
\text { número de blogs educativos, pero no es posible crearlos. Se ha de- } \\
\text { sarrollado un gran esfuerzo en este portal pero no adopta filosofías y } \\
\text { tecnologías } 2.0 \text { (sólo RSS). El usuario no participa en la construcción. }\end{array}$ \\
\hline $\begin{array}{l}\text { educarioja.org } \\
\text { (La Rioja) }\end{array}$ & $4-12,90 \%$ & $\begin{array}{l}\text { Portal educativo-formativo sin apenas presencia } 2.0 \text { (sólo RSS). El } \\
\text { usuario no participa en la construcción, tan sólo consulta y descarga. } \\
\text { La mayor parte de las características del portal están más cerca de la } \\
\text { web tradicional que de la web 2.0. }\end{array}$ \\
\hline $\begin{array}{l}\text { Educacyl } \\
\text { (Castilla y León) }\end{array}$ & $4-12,90 \%$ & $\begin{array}{l}\text { Portal informativo con pocos elementos 2.0. La participación del } \\
\text { usuario en su construcción es inexistente, su experiencia se limita a } \\
\text { consultas. Está más próximo a la web tradicional que a la web 2.0. }\end{array}$ \\
\hline $\begin{array}{l}\text { Espazo Abalar } \\
\text { (Galicia) }\end{array}$ & $2-6,45 \%$ & $\begin{array}{l}\text { Portal educativo-formativo con poca presencia 2.0. Sólo ofrece sus- } \\
\text { cripción RSS con marcadores dinámicos y aplicaciones. El resto de } \\
\text { características son tradicionales. No apuesta por la construcción co- } \\
\text { laborativa del mismo. }\end{array}$ \\
\hline Portal de Ceuta & 0 & $\begin{array}{l}\text { Realmente lo analizado no es un portal, se trata de un directorio del } \\
\text { sitio web "ceutadigital". En la evaluación, se hace rápidamente visible } \\
\text { que no contiene ninguna de las ideas ni tecnologías propias de la web } \\
\text { 2.0. }\end{array}$ \\
\hline Portal de Melilla & 0 & $\begin{array}{l}\text { En la evaluación se ve que no contiene ninguna idea ni tecnología 2,0. } \\
\text { Se trata de un portal tradicional, donde el usuario no tiene mecanis- } \\
\text { mos de participar en la construcción colaborativa del sitio web. }\end{array}$ \\
\hline
\end{tabular}


Si establecemos cuatro categorías de portales en función del número de elementos 2.0 que incorporan (ninguno; hasta 10; de 11 a 20 y de 21 a 31), se obtendría el agrupamiento que se muestra en el siguiente gráfico como resultado:

\section{Presencia 2.0 en portales educativos}

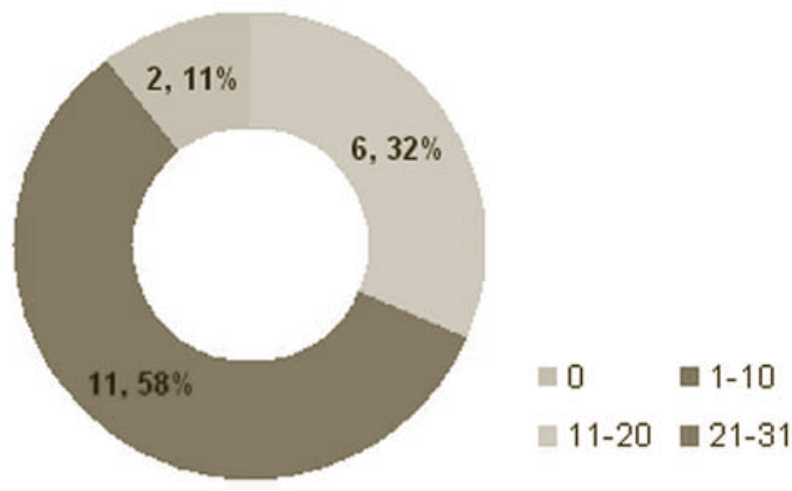

Imagen 7. Categorías de desarrollo de los portales educativos en función del número de servicios y productos 2.0 que integran. Fuente: elaboración propia a partir de la guía de evaluación

Se observa fácilmente que ninguno de los portales analizados alcanza la máxima categoría y que es abrumadoramente mayor el número de portales que están en una fase primaria de desarrollo. Otra serie de resultados destacables son los siguientes:

- Los portales educativos formativos EDUCAMADRID (Madrid) y EDU365.cat (Cataluña) son los portales mejor valorados en cuanto a número de elementos 2.0 que incorporan. En concreto incorporan 17 de 31 , lo que hace que incorporen un 58,84\% del total de servicios y productos que hemos analizado. Este valor obtenido por los portales más desarrollados demuestra la introducción de nivel intermedia de la Web 2.0 en este entorno.

- El servicio o producto 2.0 que más incorporan los portales educativos es la sindicación RSS, mediante agregadores/lectores de contenido, presentes en quince de diecinueve portales, lo que supone un 78,95\% del total de portales estudiados. Esto también puede resultar lógico porque la sindicación es un producto básico de los portales gestionados de forma dinámica con sistemas gestores de contenido.

- El etiquetado social (a través de marcadores sociales y tag clouds) son elementos 2.0 también con cierta presencia. Esto ocurre porque la 
mayoría de los portales participan en el proyecto "agrega" (federación de repositorios de objetos digitales educativos de las comunidades autónomas). El etiquetado está presente en un 73,68\% de los portales y las nubes de etiquetas en un 68,42\%. El desarrollo de etiquetas y folksonomías, típicas de la Web 2.0 comienza a tomar cuerpo en este contexto.

\section{Conclusiones}

A nivel general podemos concluir sin lugar a duda alguna que los resultados obtenidos en esta revisión no son alentadores. El camino por recorrer es aún largo, aunque se puede considerar que los portales educativos de las comunidades autónomas han realizado un gran esfuerzo por incorporar elementos más sociales y participativos en su oferta. Es también cierto que el punto de partida de los portales no es el mismo, ya que algunos aún prácticamente no han terminado de desarrollar el concepto de portal y se encuentran muy lejos aún de otras comunidades que llevan varios años trabajando con ellos. Este hecho es el que provoca las grandes desigualdades que se pueden apreciar en las tablas de resultados anteriores.

Resulta paradójico y ciertamente difícilmente explicable la no presencia de ninguno de estos servicios o productos en la oferta de los portales analizados: podcasting de video (videoblogs), redes sociales profesionales, marcadores sociales basados en almacenamientos de URL, comunidades móviles y de acceso y las aplicaciones sobre mapas. Estos elementos no aparecen en ninguno de los diecinueve portales investigados. No todos ellos resultan de una complicada implantación y la verdad es que se está desaprovechando el potencial educativo de sus formatos,

Otra evidencia de este estudio es que los portales educativos se siguen dividiendo en tres categorías: (a) los portales educativos institucionales que contienen información de carácter administrativo y de gestión educativa, de interés general para el ciudadano; (2) los portales educativos informativos, donde se ofrece información de interés general para la comunidad educativa, pero siguen incorporando información institucional o administrativa y, por último (3) están los portales educativos formativos, donde la información se encuentra más orientada hacia docentes, ofreciéndose normalmente recursos didácticos y materiales y sitios donde alojar webs, cursos, etc. Es sobre este último grupo donde se deberían concentrar los esfuerzos de inserción y establecimiento de servicios 2.0 para fomentar las actividades de aprendizaje colaborativo y el fomento de proyectos de inteligencia colectiva entre los profesores y los estudiantes. Los portales de tipo informativo-administrativo 
pueden seguir funcionando perfectamente con una escasa presencia de la Web 2.0 en ellos.

Por tanto queda trabajo por realizar y si bien la comunidad educativa parece volcada en este empeño y participa activamente de la filosofía Web 2.0, va siendo hora de que encuentre apoyo de sus administraciones, para la búsqueda de las sinergias positivas de esta innovadora tecnología con el grueso de las actividades docentes. No se trata ya de desarrollar costosos proyectos emblemáticos, que la mayoría de las veces quedan a medias, ahora se trata de adoptar una nueva filosofía de trabajo y cooperación, y de poner los medios necesarios para ello, que no son tantos.

\section{ReFERENCIAS}

Área Moreira, M. "De los webs educativos al material didáctico web", en Comunicación y Pedagogía, 188, pp.32-38, recuperado 16-03-2011 dehttp://webpages.ull.es/users/manarea/Documentos/sitiosweb.pdf.

Bedriñana Ascarza, A. (2005), "Técnicas e indicadores para la evaluación de portales educativos en Internet", en Gestión en el Tercer Milenio, Rev. de Investigación de la Facultad de Ciencias Administrativas, vol. 7, núm. 14, pp.81-88, recuperado 16-03-2011 de http://sisbib.unmsm.edu.pe/bibvirtualdata/publicaciones/administracion/n14_2005/a09.pdf.

Berners-Lee, T. (1989), Information Management: a Proposal, recuperado 25-03-2011 de http://www.w3.org/History/1989/propo sal.html.

Fundación Orange (2007), Mapa visual de la web 2.0, recuperado 2103-2011 de http://internality.com/web20/.

Lara, T. (2008), "Blogs y wikis: diferencias significativas", recuperado 1-02-2012 de http://tiscar.com/2008/04/06/blogs-y-wikis-diferen cias-significativas/.

López Carreño, R. (2004), “Análisis taxonómico de los portales periodísticos españoles”, en Anales de Documentación, núm. 7, pp. 123-140, recuperado 18-02-2011 de http://revistas.um.es/anales doc/article/view/1671/1721.

López Carreño, R. (2007), "Los portales educativos: clasificación y componentes", en Anales de Documentación, núm. 10, pp. 233 244, recuperado 08-01-2011 de http://revistas.um.es/analesdoc/ article/view/1171.

O'Reilly, T. (2005), What it Web 2.0: Design patterns and bussiness models for the next generation of software, recuperado 01-02-2012 de http://oreilly.com/web2/archive/what-is-web-20.html.

Wheeler, S. (2009), Teaching with Twister, Post publicado en el blog Learning with 'e's. y archivado en http://steve-wheeler.blogspot. com/2009/01/teaching-with-twitter.html. 
